\title{
Numerical approach to the parallel gradient operator in tokamak scrape-off layer turbulence simulations and application to the GBS code
}

\author{
S. Jolliet*, F.D. Halpern, J. Loizu, A. Mosetto, F. Riva, P. Ricci \\ École Polytechnique Fédérale de Lausanne (EPFL), Centre de Recherches en Physique des Plasmas, CH-1015 Lausanne, Switzerland
}

\section{A R T I C L E I N F O}

\section{Article history:}

Received 3 February 2014

Received in revised form

30 September 2014

Accepted 25 October 2014

Available online 8 November 2014

\section{Keywords:}

Plasma turbulence

Shear-Alfvén waves

Scrape-off layer

\begin{abstract}
A B S T R A C T
This paper presents two discretisation schemes for the parallel gradient operator used in scrape-off layer plasma turbulence simulations. First, a simple model describing the propagation of electrostatic shearAlfvén waves, and retaining the key elements of the parallel dynamics, is used to test the accuracy of the different schemes against analytical predictions. The most promising scheme is then tested in simulations of limited scrape-off layer turbulence with the flux-driven 3D fluid code GBS (Ricci et al., 2012): the new approach is successfully benchmarked against the original parallel gradient discretisation implemented in GBS. Finally, GBS simulations using a radially varying safety profile, which were inapplicable with the original scheme are carried out for the first time: the well-known stabilisation of resistive ballooning modes at negative magnetic shear is recovered. The main conclusion of this paper is that a simple approach to the parallel gradient, namely centred finite differences in the poloidal and toroidal direction, is able to simulate scrape-off layer turbulence provided that a higher resolution and higher convergence order are used.
\end{abstract}

(C) 2014 Elsevier B.V. All rights reserved.

\section{Introduction}

A magnetically confined fusion plasma is composed of a closed field line region, inside which fusion reactions take place, and an open field line region called the Scrape-Off Layer (SOL). In this narrow region the magnetic field lines terminate on either a limiter or a divertor. The physical understanding of the SOL is crucial to determine the performance of future fusion devices such as ITER as the SOL, e.g., exhausts the tokamak power, controls the plasma fuelling and the impurity dynamics. More precisely, turbulent phenomena inside the SOL have a direct influence on its width and on the power density reaching the vacuum vessel components.

Realistic turbulence simulations of the SOL are extremely challenging for several reasons. First, the SOL is characterised by background gradient lengths comparable to the typical size of turbulence, making the separation between the equilibrium and the fluctuations inconvenient. Second, the typical size of turbulent structures, of the order of the ion sound gyroradius, is several orders of magnitude smaller than the domain size. Third, the very

\footnotetext{
* Corresponding author.

E-mail address: sebastien.jolliet@epfl.ch (S. Jolliet).
}

complex magnetic geometry implies non-periodic boundary conditions and a fine grid resolution to solve for X points. Fourth, the main instabilities such as drift waves and ballooning modes are destabilised by the parallel dynamics of the electrons, while the turbulence time scale is set by the ions: the electron to ion time scale scales as $\sqrt{m_{i} / m_{e}} \sim 60$ for a Deuterium plasma. Finally, the turbulence, mostly aligned parallel to the magnetic field lines, is strongly anisotropic.

Despite the rapid increase of computational power, it remains difficult to include all the key physics ingredients in a selfconsistent manner. Although some effort to perform massively parallel kinetic simulations in the tokamak edge has started [1-3], the computational challenges remain tremendous. In fact, as several key physical mechanisms are not understood yet, most of the present studies focus on fluid models that retain the main characteristics of the SOL turbulence. The fluid approach is justified by the high collisionality in the SOL. While the first studies focused on 2D fluid turbulence, assuming $k_{\|}=0$ and modelling sheath losses [4,5], recently state-of-the art 3D fluid simulations have been developed, using a flux-driven model where profiles are $a$ priori unknown.

One of the key numerical challenges to simulate 3D turbulence in tokamaks is the accurate computation of the parallel gradient. The most popular approach is to take advantage of the strong 
anisotropy of the turbulence by using field-aligned coordinates [6-9]. The first local gyrofluid simulations using a flux tube geometry were performed in the early 90s [6]. The shifted metric [10] procedure further allowed global simulations in field-aligned coordinates. An alternative approach is to use the straight-field-line angle [11] and to filter large $k_{\|}$modes [12]. Unfortunately, these two approaches are inapplicable in several situations such as large electromagnetic perturbations, time-dependent magnetic fields and $\mathrm{X}$-point geometry, for which the safety factor goes to infinity and both the straight-field-line and the field-aligned coordinate become singular.

Recently, a new approach called the Flux Coordinates Independent (FCI) approach has been proposed [13], in which the fluid quantities of interests are interpolated along the field-line using cubic Hermite interpolation. However, the FCI method has been tested for closed field line configuration only. Additionally, several magnetohydrodynamic and turbulence studies have been performed without taking advantage of field-aligned property [14-17]. Although the required resolution in such cases is higher compared to field-aligned methods, the implementation is usually simpler. An additional difficulty specific to the SOL region comes from the non-periodic boundary conditions. In proximity of a divertor or a limiter, the parallel gradient scheme must be modified, for example, by using one-sided derivatives [17].

The aim of this work is to test the implementation of a nonfield-aligned approach to simulate tokamak SOL turbulence using GBS, a 3D global fluid flux-driven code [18]. GBS was specifically conceived to study plasma turbulence in settings where the high fluctuation level and the large radial and poloidal mode extension prevent the expansion of the model equations by separating turbulent and equilibrium length scales. Until now, GBS has employed a field-aligned parallel gradient operator.

Studies carried out using GBS simulations have helped provide an understanding of the turbulence saturation mechanisms in the SOL, the non-linear turbulent regimes, the scaling of the tokamak SOL width, the role of electromagnetic effects, and the equilibrium electric field [19-22]. Since the code was designed around a simple, robust, and scalable numerical scheme, we are able to simulate the SOL of medium size tokamaks such as TCV or Alcator C-Mod at a cost of roughly $10^{6} \mathrm{CPU}$ hours on the Helios supercomputer at the International Fusion Energy Research Center, based on Intel Sandy Bridge processors.

The main conclusion of our study is that SOL turbulence simulations using a non-field aligned approach are still possible at a reasonable cost, within an algorithm that can be extended to simulate more complex magnetic geometries. Using standard centred finite difference formulas for the parallel gradient operator, it was found that it is possible to reproduce the results of the field-aligned with an increase in computational cost of roughly $10 \%$. This modest increase in cost results from mutually cancelling changes in resolution requirements: the number of toroidal grid points must be doubled in order to achieve the required resolution and stability, but the number of poloidal grid points can be decreased by a factor of two.

Earlier GBS simulations described in the literature were carried out using a centred, field-aligned finite difference scheme for the parallel gradients. Fluid quantities are evaluated on a nonfield aligned grid with their parallel gradient being computed by appropriately choosing the grid resolution such that the field lines intersect grid points exactly. This approach is limiting for several reasons, the main one being its inapplicability to treat radially varying safety factor profiles, and, therefore, $X$ points. In this paper, the simplest parallel gradient operator that should be able to treat this configuration, i.e. computing separately the derivatives along the poloidal and toroidal directions, is tested. First, its numerical properties are studied on the propagation of shear-Alfvén waves
(SAWs). The necessary requirements for a satisfactory accuracy are obtained by comparing the analytical dispersion relation with the numerical results. Then, the new numerical scheme is tested in GBS nonlinear simulations. Benchmark between simulations using the field-aligned and the non-field-aligned parallel gradient operator show that the latter is able to capture SOL turbulence correctly. Finally, a physical example is given by considering a radially varying safety factor profile.

This paper is organised as follows. The fluid model used to study SOL physics and employed by GBS is introduced in Section 2. The details of the parallel gradient operator schemes (both the one that has been used by GBS in the past and various proposed ones) are derived in Section 3. These numerical methods are applied on the analytical test case of the SAWs in Section 4. Nonlinear GBS simulations are then presented in Section 5. Finally, the conclusions are given in Section 6.

\section{Fluid model of the SOL plasma}

The study of the SOL presented in this paper is based on the two-fluid, electromagnetic, cold ion $\left(T_{i}=0\right)$ drift-reduced Braginskii equations. By assuming the ordering $\mathrm{d} / \mathrm{d} t \ll \omega_{c i}\left(\omega_{c i}=e B / m_{i}\right.$ is the ion gyrofrequency) and $k_{\perp} \gg k_{\|}$, the perpendicular velocities are written as $\mathbf{V}_{\perp i}=\mathbf{V}_{E \times B}+\mathbf{V}_{\text {pol }}$ and $\mathbf{V}_{\perp e}=\mathbf{V}_{E \times B}+\mathbf{V}_{* e}$, where $\mathbf{V}_{E \times B}=(-\nabla \phi \times \mathbf{B}) / B$ is the $E \times B$ drift velocity, $V_{* e}=$ $-\left(1 / e n B^{2}\right) \mathbf{B} \times \nabla p_{e}$ is the electron diamagnetic drift, $e$ is the elementary charge, and $\mathbf{V}_{\text {pol }}$ is the polarisation velocity defined in Ref. [23]. The continuity, vorticity, ion and electron parallel momentum and electron temperature equations then read, in normalised form:

$$
\begin{aligned}
& \partial_{t} n=-\frac{R_{0}}{B_{0}}[\phi, n]-\nabla_{\|}\left(n v_{\| e}\right)-n v_{\| e} \nabla \cdot \mathbf{b} \\
& +\frac{2}{B_{0}}\left[C\left(p_{e}\right)-n C(\phi)\right]+S_{n}+D_{n} \nabla_{\perp}^{2} n \\
& \partial_{t} \omega=-\frac{R_{0}}{B_{0}}[\phi, \omega]-v_{\| i} \nabla_{\|} \omega+\frac{2 B_{0}}{n} C\left(p_{e}\right) \\
& +B_{0}^{2}\left[\frac{1}{n} \nabla_{\|} j_{\|}+\frac{j_{\|}}{n} \nabla \cdot \mathbf{b}\right] \\
& +\frac{B_{0}}{3 n} C\left(G_{i}\right)+D_{\omega} \nabla_{\perp}^{2} \omega \\
& \partial_{t} \chi=-\frac{R_{0}}{B_{0}}\left[\phi, v_{\| e}\right]-v_{\| e} \nabla_{\|} v_{\| e}+D_{v_{\| e}} \nabla_{\perp}^{2} v_{\| e} \\
& +\frac{m_{i}}{m_{e}}\left\{v \frac{j_{\|}}{n}+\nabla_{\|} \phi-\frac{1}{n} \nabla_{\|} p_{e}\right. \\
& \left.-0.71 n \nabla_{\|} T_{e}-\frac{2}{3 n} \nabla_{\|} G_{e}\right\} \\
& \partial_{t} v_{\| i}=-\frac{R_{0}}{B_{0}}\left[\phi, v_{\| i}\right]-v_{\| i} \nabla_{\|} v_{\| i}-\frac{1}{n} \nabla_{\|} p_{e} \\
& -\frac{2}{3 n} \nabla_{\|} G_{i}+D_{v_{\| i}} \nabla_{\perp}^{2} v_{\| i} \\
& \partial_{t} T_{e}=-\frac{R_{0}}{B_{0}}\left[\phi, T_{e}\right]-v_{\| e} \nabla_{\|} T_{e} \\
& +\frac{4}{3 B_{0}} T_{e}\left[\frac{7}{2} C\left(T_{e}\right)+\frac{T_{e}}{n} C(n)-C(\phi)\right] \\
& +S_{T_{e}}+\frac{2}{3} T_{e}\left[0.71 \nabla_{\|} v_{\| i}-1.71 \nabla_{\|} v_{\| e}\right. \\
& \left.+0.71\left(\frac{v_{\| i}-v_{\| e}}{n}\right) \nabla_{\|} n\right] \\
& +T_{e}\left(0.71 v_{\| e}-1.71 v_{\mid i}\right) \nabla \cdot \mathbf{b}+D_{T_{e}} \nabla_{\perp}^{2} T_{e}+\chi_{\|} \nabla_{\|}^{2} T_{e},
\end{aligned}
$$

where $R_{0}$ is the tokamak major radius expressed in $\rho_{s 0}$ units, $\omega=$ $\nabla_{\perp}^{2} \phi$ is the vorticity, $j_{\|}=n\left(v_{\| i}-v_{\| e}\right)$ is the parallel current, 
$\mathbf{b}_{\mathbf{0}}=\mathbf{B}_{\mathbf{0}} / B_{0}$ is the unit unperturbed magnetic field vector, $\chi=$ $v_{\| e}+m_{i} \beta_{e 0} \psi /\left(2 m_{e}\right), \beta_{e 0}=2 \mu_{0} p_{e} / B_{0}^{2}$ is the plasma beta and $\psi$ is such that $-\beta_{e 0} \psi / 2=-\mathbf{b} \cdot \mathbf{A}_{1}$ where $\mathbf{A}_{1}$ is a vector potential function such that $\nabla \times \mathbf{A}_{1}=\mathbf{B}_{1}$. The normalised Spitzer resistivity is $v$, and $\chi_{\|}$is the parallel heat flux diffusivity given as an input constant. Quasineutrality is assumed such that $n_{e}=n_{i} \equiv n$. Plasma outflow from the closed flux surface region is mimicked using density and temperature sources, respectively, $S_{n}$ and $S_{T_{e}}$. The $G_{e}$ and $G_{i}$ terms represent the gyroviscous part of the pressure tensor and are given by:

$G_{i}=-3 \eta_{0 i}\left[\frac{2}{3} \nabla_{\|} v_{\| i}+\frac{1}{3 B_{0}} C(\phi)\right]$

$G_{e}=-3 \eta_{0 e}\left[\frac{2}{3} \nabla_{\|} v_{\| e}+\frac{1}{3 B_{0}} C(\phi)-\frac{1}{3 B_{0} n} C\left(p_{e}\right)\right]$

where $\eta_{0 i}$ and $\eta_{0 e}$ are constant coefficients given on input. The gyroviscous coefficient $\eta_{0 i}$ is formally equal to 0 in a cold ion model, but a finite value is used for numerical stability; the value of $\eta_{0 e}$ used in GBS is usually larger than the physical one but it has been checked not to influence the main results presented herein. Furthermore, the vorticity equation has been obtained using the commonly used Boussinesq approximation:

$\nabla \cdot\left(\frac{n m_{i}}{B_{0}^{2} e} \frac{\mathrm{d}}{\mathrm{d} t} \nabla_{\perp} \phi\right) \cong \frac{n m_{i}}{B_{0}^{2} e} \frac{\mathrm{d}}{\mathrm{d} t} \nabla_{\perp}^{2} \phi$.

This approximation is equivalent to neglecting the space dependence of $n / B_{0}^{2}$ in the polarisation current and to assume that the $\mathrm{d} / \mathrm{d} t$ and $\nabla$ operators commute.

Small perpendicular diffusion terms of the form $D_{a} \nabla_{\perp}^{2} a$ are added for numerical reasons. Perpendicular Laplacians are assumed to lie in the poloidal plane. The curvature operator is defined by $C(A)=B_{0} / 2\left[\nabla \times\left(\mathbf{b}_{0} / B_{0}\right)\right] \cdot \nabla A$, the parallel gradient is $\nabla_{\|} A=\mathbf{b}_{0} \cdot \nabla A+\beta_{e 0} R_{0}[\psi, A]$, the perpendicular Laplacian is $\nabla_{\perp}^{2} A=-\nabla \cdot\left[\mathbf{b}_{0} \times\left(\mathbf{b}_{0} \times \nabla A\right)\right]$ and the Poisson bracket is $[\phi, A]=$ $\mathbf{b}_{0} \cdot(\nabla \phi \times \nabla A)$. The differential operators are written for a general geometry:

$[\phi, A]=\frac{1}{g} \epsilon_{i j k} b_{0 i} \frac{\partial \phi}{\partial \xi^{j}} \frac{\partial A}{\partial \xi^{k}}$

$\nabla_{\|} A=b^{0 j} \frac{\partial A}{\partial \xi^{j}}$

$\nabla_{\|}^{2} A=b^{0 i} b^{0 j} \frac{\partial^{2} A}{\partial \xi^{j} \partial \xi^{i}}+b^{0 i} \frac{\partial b^{0 j}}{\partial \xi^{i}} \frac{\partial A}{\partial \xi^{j}}$

$C(A)=\frac{B_{0}}{2 \mathcal{g}} \frac{\partial c_{m}}{\partial \xi^{j}} \frac{\partial A}{\partial \xi^{k}} \epsilon_{k j m}$

$\nabla_{\perp}^{2} A=\frac{1}{g} \frac{\partial}{\partial \xi^{k}}\left(g^{-1} \epsilon_{k l m} \epsilon_{i \alpha \beta} g_{m i} b_{0 l} b_{0 \alpha} \frac{\partial A}{\partial \xi^{\beta}}\right)$

$\nabla \cdot \mathbf{b}=\frac{1}{g} \frac{\partial}{\partial \xi^{i}}\left(b^{0 i} g\right)$

$B_{0}=\sqrt{B^{0 j} B_{0 j}}$

Here, repeated indices are summed over using the usual Einstein notation, vectors have been expanded using their co or contravariant basis, and $g=\left[\nabla \xi^{1} \cdot\left(\nabla \xi^{2} \times \nabla \xi^{3}\right)\right]^{-1}$ is the Jacobian.

The normalisations are (tilde denotes quantities in physical units): $t=\tilde{t} /\left(R_{0} / c_{s 0}\right), \nabla_{\perp}=\tilde{\nabla}_{\perp} \rho_{s 0}, \nabla_{\|}=\tilde{\nabla}_{\|} R_{0}, v=\tilde{v} / c_{s 0}$, $n=\tilde{n} / n_{0}, T_{e}=\tilde{T}_{e} / T_{e 0}, \phi=e \tilde{\phi} / T_{e 0}, v=\tilde{v} /\left(c_{s 0} m_{e} / R_{0} m_{i}\right)$, with $c_{s 0}=\sqrt{T_{e 0} / m_{i}}$ and $\rho_{s 0}=c_{s 0} / \omega_{c i}$, while $T_{e 0}$ and $n_{0}$ are the reference temperature and density.
The above system of equations is completed by a proper set of boundary conditions to describe the interface between the SOL and the magnetic pre-sheath where the ion drift approximation $\mathrm{d} / \mathrm{d} t \ll \omega_{c i}$ breaks down [24]. At the top and bottom of the limiter, these are, in normalised units, $v_{\| i}= \pm \sqrt{T_{e}}, v_{\| e}=$ $\pm \sqrt{T_{e}} \exp \left(\Lambda-\phi / T_{e}\right), \partial_{s} n=\mp n / \sqrt{T_{e}} \partial_{s} v_{\| i}, \partial_{s} \phi=\mp \sqrt{T_{e}} \partial_{s} v_{\| i}$, $\omega=-\left(\partial_{s} v_{\| i}\right)^{2} \mp \sqrt{T_{e}} \partial_{s s} v_{\| i}, \psi=0$ and $\partial_{s} T_{e}=\kappa_{T} / T_{e} \partial_{s} \phi$, with $\Lambda=3$ and $\kappa_{T} \cong 0.15$. Here $s$ is a coordinate normal to the limiter walls. Corrections of order $\rho_{s 0} / L_{p}$ described in Ref. [24] have been neglected.

The radial boundary conditions, which are not described in Ref. [24], can be specified as either Dirichlet or Neumann. Typically, we specify $\partial_{r} n=\partial_{r} T_{e}=\partial_{r} \chi=\partial_{r} v_{\| i}=0$ and $\omega=0$ at the radial boundaries. Since the electrostatic potential is tightly coupled to the temperature [22], we use $\phi=\Lambda T_{e}$ as a radial boundary condition. Finally, as is customary in flux-driven codes, a small buffer region (roughly, 10 grid points wide) is implemented in order to decrease the amplitude of the fluctuations as they approach the simulation boundary.

The system of equations (1)-(5) with the boundary conditions described above has been implemented in the GBS code. These equations are general, and can be formulated in an arbitrary coordinate system. Note that similar equations are implemented in other edge and SOL turbulence codes [25,17,26-29]. In GBS, $\left(x=r, y=a \theta_{*}, z=R_{0} \varphi\right.$ ) is used, where $\varphi$ is the toroidal angle and $\theta_{*}$ is the straight-field-line coordinate defined by:

$\theta_{*}=\frac{1}{q(r)} \int_{0}^{\theta} \mathrm{d} \theta^{\prime} \frac{\mathbf{B}_{0} \cdot \nabla \varphi}{\mathbf{B}_{0} \cdot \nabla \theta^{\prime}}$

$q(r)=\frac{1}{2 \pi} \int_{0}^{2 \pi} \mathrm{d} \theta \frac{\mathbf{B}_{0} \cdot \nabla \varphi}{\mathbf{B}_{0} \cdot \nabla \theta}$

where $q(r)$ is the safety factor. The straight-field-line coordinate is such that $\mathbf{B}_{0} \cdot \nabla \theta_{*}=\mathbf{B}_{0} \cdot \nabla \varphi$. In other words, the magnetic field lines are straight in the $(y, z)$ plane.

Derivatives in the $x$ and $y$ directions, needed to compute the curvature, Laplacian and diffusion operators, are computed using standard second order centred finite difference schemes, while the Poisson bracket operator is discretised using the Arakawa scheme [30]. This implies that one ghost cell must be added in the $x$ and $y$ directions at the boundaries. The parallel gradient scheme is detailed in Section 3. The integration is done with an explicit 4th order Runge-Kutta scheme. At each substep, the boundary conditions presented in Section 2 are imposed and the potential is obtained by inverting the linear system $\nabla_{\perp}^{2} \phi=\omega$.

GBS is implemented using standard MPI domain decomposition in the $x$ and $z$ direction. At each iteration substep, the ghost cells are communicated to their neighbouring processors in both $x$ and $z$ directions. The Poisson operator is independent of $z$, which naturally leads to the parallelisation of the Poisson solver in the $z$ direction. The linear system is solved using the parallel direct solver MUMPS with a domain decomposition along the $x$ communicator.

The parallel dynamics plays a crucial role in SOL physics. Plasma is transported parallel to the field lines with a velocity much larger than in the perpendicular direction and it is lost at the limiter. This plasma parallel flow requires an accurate description of the parallel gradient, which should not lead to unphysical flow in the perpendicular directions. Then, the parallel dynamics allows $k_{\|} \neq$ 0 modes such as Drift Waves to develop when the adiabaticity of electrons is broken. Finally, large $k_{\|}$modes are usually damped and will influence the turbulence spectrum: an inaccurate formulation of the parallel gradient operator may lead to spurious modes and spectral pollution. 

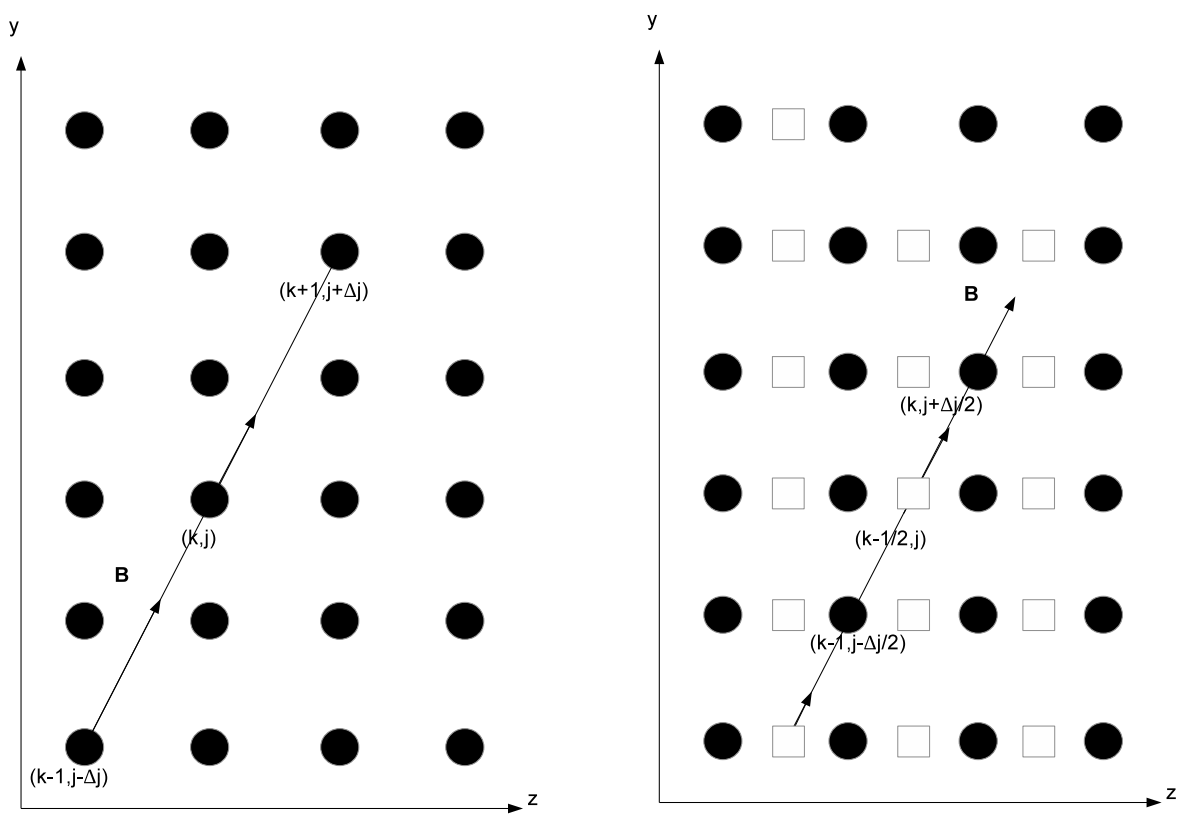

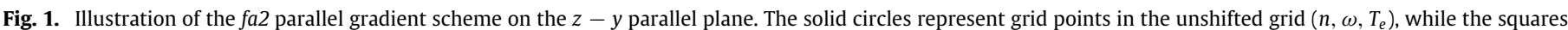

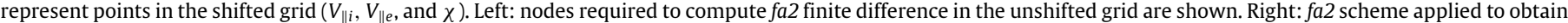
the parallel gradient of a quantity defined in the shifted grid.

\section{Parallel gradient schemes}

In this section, the various schemes that can be used to compute the first and second order parallel gradient operators are given. For instance, the $y$ direction has $N_{y}$ grid points, numbered from 1 to $N_{y}$, the ghost cells being indexed with 0 and $N_{y}+1$. The $z$ coordinate is periodic, and ghost cells are necessary at each end the domaindecomposed $z$ grid in order to compute the parallel gradients. To evaluate centred finite difference schemes, the number of ghost cells is increased as the order of the scheme increases.

\subsection{The field-aligned parallel gradient scheme}

The property of straight field lines in the $(y, z)$ plane has been used in the past by GBS to compute the parallel gradient in a fieldaligned way. The concept is illustrated in Fig. 1. If the number of grid intervals in the $y$ and $z$ directions are conveniently chosen, the field lines will intersect the grid points and the parallel gradient can be computed using a field-aligned second-order finite difference scheme, named hereafter as the fa2 scheme:

$$
\begin{aligned}
\left.\nabla_{\| 0} A\right|_{i, j, k} & =q b^{\theta *} R_{0} \frac{\partial A}{\partial \zeta} \\
& =q b^{\theta *} R_{0} \frac{1}{2 \Delta \zeta}\left(A_{i, j+\Delta j, k+1}-A_{i, j-\Delta j, k-1}\right)
\end{aligned}
$$

where $\zeta=z+a y / q$ is a field-following coordinate, $\Delta \zeta=$ $\Delta z \sqrt{1+\epsilon^{2} / q^{2}}, \epsilon=a / R_{0}$ is the inverse aspect ratio, $\Delta j=N_{y} /$ $\left(q N_{z}\right)$ and $N_{y}$ (respectively $N_{z}$ ) are the number of grid points in the $y$ (respectively $z$ ) direction. The second order parallel gradient is approximated as:

$$
\begin{aligned}
& \left.\nabla_{\| 0}^{2} A\right|_{i, j, k}=\left(q b^{\theta *} R_{0}\right)^{2} \frac{\partial^{2} A}{\partial \zeta^{2}} \\
& =\left(q b^{\theta *} R_{0}\right)^{2} \frac{1}{(\Delta \zeta)^{2}}\left(A_{i, j+\Delta j, k+1}-2 A_{i, j, k}+A_{i, j-\Delta j, k-1}\right)
\end{aligned}
$$

and a small term proportional to $\partial\left(q b^{\theta *} R_{0}\right) / \partial \zeta$ has been neglected as it is usually extremely small.
In expressions (18) and (19), $\Delta j=N_{y} /\left(q N_{z}\right)$ is constrained to be an integer. Furthermore, to avoid a null-space problem, a staggered grid in the $z$ direction, shifted by one half cell to the left, must be used. More precisely, in GBS, $n, \omega, \phi$, and $T_{e}$ are expressed on the standard (unshifted) grid while $v_{\| e}$ and $v_{\| i}$ are discretised on the shifted grid. The parallel gradient must therefore be carefully computed when one needs the gradient of a field discretised on a shifted grid on an unshifted grid, and vice-versa. The parallel gradient becomes:

$$
\begin{aligned}
\left.\nabla_{\| 0}^{u \rightarrow s} A\right|_{i, j, k} & =q b^{\theta_{*}} R_{0} \frac{\partial A}{\partial \zeta} \\
& =q b^{\theta_{*}} R_{0} \frac{1}{2 \Delta \zeta}\left(A_{i, j+\Delta j / 2, k}-A_{i, j-\Delta j / 2, k-1}\right) .
\end{aligned}
$$

Consequently, $\Delta j$ is constrained to be an even integer. Simulations with odd $\Delta j$ could be obtained by further shifting by one poloidal half cell the shifted z-grid; this has not been implemented in GBS. Special care must be taken at the limiter. In fact, for example, it is impossible to compute the parallel gradient at the first and last poloidal point $j=1$ and $j=N_{y}$ using Eq. (18) as the grid points $j=1-\Delta j$ and $j=N_{y}+\Delta j$ do not exist. The parallel gradient is therefore computed using a 3 points stencil one-sided derivative.

For the second order parallel gradients, the one-sided derivative scheme has been found unstable. Instead, a partially centred scheme, represented in Fig. 2, is used. The field line intersects the $j=N_{y}+1$ line at a position $k+\delta$ depending on the safety factor. Since $\Delta j-1$ points require a partially centred scheme, the values of $\delta$ in ]0, 1 [ are given by $l / \Delta j, l=1, \ldots, \Delta j$ when the second order parallel gradient is evaluated at $j=N_{y}-\Delta j+2, \ldots, N_{y}$. In fact, the second order parallel gradient for $j=N_{y}-\Delta j+2, \ldots, N_{y}$ becomes:

$$
\begin{aligned}
& \left.\nabla_{\| 0}^{2}\right|_{i, j, k}=\frac{\left(q b^{\theta *} R_{0}\right)^{2}}{\Delta \zeta^{2}}\left(a_{\delta} A_{i, N_{y}+1, k+\delta}+b_{\delta} A_{i, j, k}+c_{\delta} A_{i, j-\Delta j, k-1}\right) \\
& a_{\delta}=\frac{2}{\delta(\delta+1)}
\end{aligned}
$$




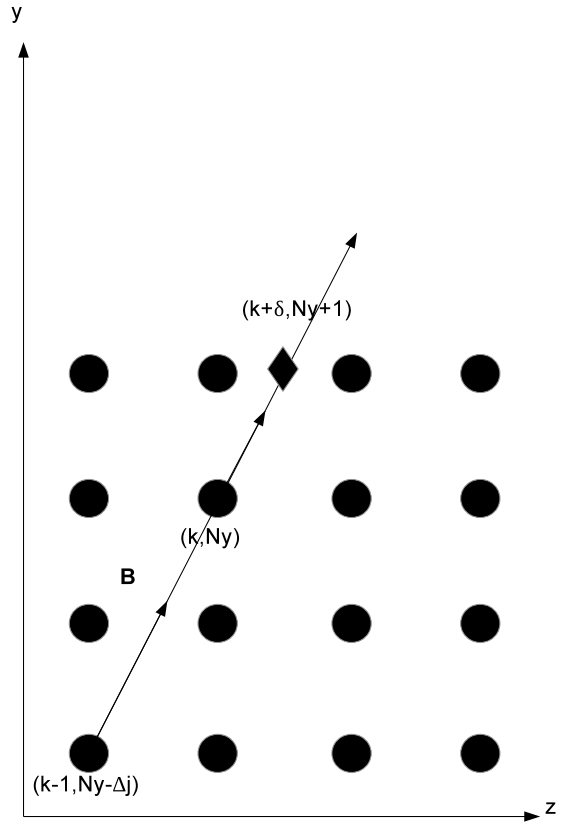

Fig. 2. Diagram of the second order parallel gradient scheme at the top edge for $\Delta j=2$. The field-line crossing the last grid point $\left(k, N_{y}\right)$ intersects the $N_{y}+1$ plane at a position $k+\delta$, with $\delta \in] 0: 1$ [ that depends on the field line pitch. The stencil is therefore composed of the three points $\left(k-1, N_{y}-1\right),\left(k, N_{y}\right)$, and $\left(k+\delta, N_{y}+1\right)$, which can be used to evaluate Eqs. (21)-(24). If $\Delta j>2$, the second order parallel gradient scheme at the grid points $\left(k, N_{y}-\Delta j+2\right),\left(k, N_{y}-\Delta j+1\right), \ldots,\left(k, N_{y}\right)$ are computed in a similar way. Equivalent expressions are used at the bottom edge.

$b_{\delta}=-\frac{2}{\delta}$

$c_{\delta}=\frac{2}{(\delta+1)}$

and the standard coefficients of the second order scheme are recovered for $\delta=1$. For $\delta \neq 1$, the value of $A_{i, N_{y}+1, k+\delta}$ is obtained through a quadratic interpolation in the $z$ direction.

Finally, it is noted that the fluid fields are interpolated between grids using 2nd order accurate parallel interpolation:

$\left.A\right|_{i, j, k+1 / 2}=\frac{1}{2}\left(\left.A\right|_{i, j+\Delta j / 2, k+1}+\left.A\right|_{i, j-\Delta j / 2, k}\right)$.

While grid shifting is not numerically reversible, interpolations of the necessary fluid quantities take place once during each evaluation of the right-hand-side of the equations. Therefore, it is not necessary to invert the interpolation to the initial grid.

The fa2 scheme has been extensively used in electrostatic and electromagnetic simulations of SOL turbulence in limited configurations [31,21,24,22,20,32] but has several limitations. First, the constraint $\Delta j \geq 2$ implies $N_{y} \geq 2 q N_{z}$. Depending on the plasma parameters, SOL turbulence is dominated by ballooning modes with $k_{\|} \approx 0$ or by drift waves with a small $k_{\|}$. It is then reasonable to assume that $N_{y} \cong q N_{z}$. The present parallel gradient scheme tends to over-resolve turbulent structures in the poloidal direction by, at least, a factor of 2 . Second, this scheme cannot be used to simulate a radially varying safety factor profile. $\Delta j$ would become a function of the radial surface and could not be maintained as an integer everywhere. Third, the fa2 scheme relies on the straight-field-line angle which is singular at an X-point. The fa2 scheme is therefore ineffective to treat realistic SOL geometries. These drawbacks motivate the development of another parallel gradient scheme.

\subsection{An alternative parallel gradient scheme for non-periodic systems}

To overcome the drawbacks exposed in Section 3, an alternative parallel gradient scheme should have the following properties: first, the scheme should not be tied to the straight-field-line coordinate and should be applicable to arbitrary coordinates. Second, the new boundary conditions at the limiter should be easily implemented in the new scheme. The real-space parallel gradient scheme is perhaps the simplest approach one can think of. The parallel gradient is simply expressed as a sum of derivatives in the $y$ and $z$ direction:

$\nabla_{\| 0} A=b^{0 y} \frac{\partial A}{\partial y}+b^{0 z} \frac{\partial A}{\partial z}$

and analogously, the second order parallel gradient is:

$$
\begin{aligned}
\nabla_{\| 0}^{2} A= & \left(b^{0 y}\right)^{2} \frac{\partial^{2} A}{\partial y^{2}}+2 b^{0 y} b^{0 z} \frac{\partial^{2} A}{\partial y \partial z}+\left(b^{0 z}\right)^{2} \frac{\partial^{2} A}{\partial z^{2}} \\
& +b^{0 y} \frac{\partial b^{0 y}}{\partial y} \frac{\partial A}{\partial y}+b^{0 y} \frac{\partial b^{0 z}}{\partial y} \frac{\partial A}{\partial z} .
\end{aligned}
$$

Both the $\partial / \partial y$ and $\partial / \partial z$ operators have been discretised with 2 nd, 4 th or 6 th order centred finite differences. These schemes will be abbreviated as the $y z 2, y z 4$ and $y z 6$ schemes. At the $y$ boundaries, partially-centred derivatives are used, using the ghost cell value at one of the extremities of the stencil. For a 4th order finite difference scheme, the first and second order $y$ derivatives at $j=1$ are given by:

$$
\begin{aligned}
& \left.\frac{\partial A}{\partial y}\right|_{j=1}=\frac{1}{12 \Delta y}\left(-3 A_{0}-10 A_{1}+18 A_{2}-6 A_{3}+A_{4}\right) \\
& \left.\frac{\partial^{2} A}{\partial y^{2}}\right|_{j=1}=\frac{1}{12 \Delta y}\left(11 A_{0}-20 A_{1}+6 A_{2}+4 A_{3}-A_{4}\right) .
\end{aligned}
$$

For a 6 th order finite difference scheme, the first and second order $y$ derivatives at $j=1,2$ are given by:

$$
\begin{aligned}
\left.\frac{\partial A}{\partial y}\right|_{j=1}= & \frac{1}{60 \Delta y}\left(-10 A_{0}-77 A_{1}+150 A_{2}-100 A_{3}\right. \\
& \left.+50 A_{4}-15 A_{5}+2 A_{6}\right) \\
\left.\frac{\partial A}{\partial y}\right|_{j=2}= & \frac{1}{60 \Delta y}\left(2 A_{0}-24 A_{1}-35 A_{2}+80 A_{3}-30 A_{4}\right. \\
& \left.+8 A_{5}-A_{6}\right) \\
\left.\frac{\partial^{2} A}{\partial y^{2}}\right|_{j=1}= & \frac{1}{180 \Delta y^{2}}\left(137 A_{0}-147 A_{1}-255 A_{2}+470 A_{3}\right. \\
& \left.-285 A_{4}+93 A_{5}-13 A_{6}\right) \\
\left.\frac{\partial^{2} A}{\partial y^{2}}\right|_{j=2}= & \frac{1}{180 \Delta y^{2}}\left(-13 A_{0}+228 A_{1}-420 A_{2}+200 A_{3}\right. \\
& \left.+15 A_{4}-12 A_{5}+2 A_{6}\right) .
\end{aligned}
$$

Similar formulas can be obtained at $j=N_{y}$ and $j=N_{y}-1$. Finally, we note that within the 4th and 6th order schemes consecutive poloidal planes are coupled through the parallel advection terms. Therefore, we have not used shifted-grids for these schemes.

\subsection{Parallel gradient scheme for periodic systems}

If the system is periodic, one can use a Fourier decomposition in the poloidal and toroidal directions. Writing $A(x, y, z)=$ $\sum_{k_{y}, k_{z}} \hat{A}_{k_{y}, k_{z}}(x) e^{i k_{y} y} e^{i k_{z} z}$, one has:

$\nabla_{\| 0} \hat{A}_{k_{y}, k_{z}}(x)=i\left(k_{y} b^{y}+k_{z} b^{z}\right) \hat{A}_{k_{y}, k_{z}}(x)$

$\nabla_{\| 0}^{2} \hat{A}_{k_{y}, k_{z}}(x)=-\left(k_{y} b^{y}+k_{z} b^{z}\right)^{2} \hat{A}_{k_{y}, k_{z}}(x)$. 
This scheme will be abbreviated as the $m n$ scheme and it will be used as a benchmark for the other operators in Section 4. This scheme has not been implemented in GBS, which features a nonpoloidal periodic geometry.

\section{Verification of the parallel gradient schemes in a shear- Alfvén wave model}

In order to test various schemes for the parallel gradient operator, we consider the minimal model containing parallel dynamics relevant to Eqs. (1)-(5), the drift-reduced Braginskii system. In the tokamak SOL, unstable modes are either driven by the compressibility of curvature-driven flows or by $\mathbf{E} \times \mathbf{B}$ convection of pressure perturbations. In both of these cases (ballooning modes and drift waves) mode growth is fed by the radial variation of density or temperature. In addition to these unstable modes, there are oscillating modes, in particular the shear-Alfén waves, which arise from the coupling of the vorticity equation (Eq. (2)) with the parallel electron dynamics described by Ohm's law (Eq. (3)). In the driftreduced model, the parallel coupling effect is affected by collisions, electron inertia, or by magnetic field fluctuations.

We implement here a standalone code describing the parallel dynamics of a simplified version of the drift-reduced Braginskii system in a single $y-z$ parallel plane. In particular, we concentrate on a model containing electrostatic shear-Alfvén waves mediated by electron inertial. The equations are given by:

$$
\begin{aligned}
& \frac{\partial v_{\| e}}{\partial t}=\frac{m_{i}}{m_{e}} \nabla_{\|} \phi+\frac{4 \eta}{3} \nabla_{\|}^{2} v_{\| e} \\
& \frac{\partial \nabla_{\perp}^{2} \phi}{\partial t}=-\nabla_{\|} v_{\| e}
\end{aligned}
$$

where $\eta=\eta_{0 e}$ and, to carry out the test in the simplest possible scenario, the domain is assumed to be poloidally and toroidally periodic. This system describes the propagation of the electrostatic SAW [33] subject to parallel diffusion and is a subset of the driftreduced equations. SAWs are encountered in both fluid and gyrokinetic simulations of electron turbulence and are high-frequency stable waves that can limit the time step when using an explicit scheme. The frequency of these waves can be decreased by adding electromagnetic perturbations or simply by neglecting electron inertia.

This model, implemented as a standalone code, represents an ideal testbed for the parallel gradient schemes for several reasons. First, it contains the key elements of a scheme involving a parallel gradient which can be tested using the same GBS numerical scheme. Second, the $k_{\|}$of the initial perturbation can be chosen in agreement to what is observed in GBS simulations and remains fixed in time, therefore simplifying the analysis of the results. Third, the SAW are the fastest waves in the full GBS system such that the time steps used in both codes are of the same order of magnitude. Fourth, results can be compared to an analytical dispersion relation, as contrary to the SOL model described in Section 2, this test case can be run in a periodic system. As a matter of fact, the proper performance of a numerical scheme for the parallel gradient in the SAW model is a necessary condition for the implementation.

The dispersion relation can be obtained assuming perturbations of the form $\exp \left[i\left(k_{y} y+k_{z} z-\omega t\right)\right]$, giving:

$\omega^{2}+2 i \gamma_{D} \omega-\omega_{0}^{2}=0$

with:

$$
\begin{aligned}
& \omega_{0}=\sqrt{\frac{m_{i}}{m_{e}}} \frac{k_{\|}}{k_{\perp}} \\
& \gamma_{D}=\frac{2 \eta}{3} k_{\|}^{2}
\end{aligned}
$$

and $k_{\|}=a / q k_{y}+k_{z}$. When parallel diffusion is absent, Eqs. (36) and (37) describe an electrostatic SAW propagating at a frequency $\omega_{0}$. Parallel diffusion introduces a damping rate $\gamma_{D}$ and decreases the real frequency to $\sqrt{\omega_{0}^{2}-\gamma_{D}^{2}}$. The mode becomes purely damped for $\eta>\eta_{c}$, where:

$\eta_{c}=\frac{3}{2} \sqrt{\frac{m_{i}}{m_{e}}} \frac{1}{k_{\|} k_{\perp}}$.

For $\eta<\eta_{c}$, the damping increases linearly with $\eta$, while for $\eta>\eta_{c}$ the damping decreases and tends towards 0 .

A 2D code simulating the SAW system has been developed using the same numerical techniques as those proposed for GBS. The simulation is initialised with a perturbation of the form $\sin (m y / a-$ $n z)$ with $m=n q+\Delta m, \Delta m=k_{\|} q$ being an input parameter that sets the parallel wave number of the mode. Typical values for $n$ and $\Delta m$ are chosen based on GBS simulations. $n$ is chosen such that $k_{y} \cong 2 \pi n q / L_{y} \cong 0.1$, value commonly observed in nonlinear steady states and $\Delta m=1-10$ is chosen based on typical GBS spectra.

The explicit time integration constrains the time step to the following CFL condition:

$\Delta t \leq \frac{4 \pi^{2}}{L_{y} N_{z}} \sqrt{\frac{m_{e}}{m_{i}}}$.

The initialised mode oscillates at a frequency and is damped at a rate that can be compared to the solution of the dispersion relation (38).

First, the accuracy of the different schemes is examined by applying the different numerical operators to the analytical perturbation $f(y, z)=\sin (m y / a-n z)$. The relative numerical error, defined by:

$E=\frac{\max _{y, z}\left[\left(\nabla_{\|} f\right)_{\mathrm{num}}-\left(\nabla_{\|} f\right)_{\mathrm{ana}}\right]}{\max _{y, z}\left(\nabla_{\|} f\right)_{\mathrm{ana}}}$,

is reported against the grid resolution in Fig. 3 for periodic and non-periodic discretisation schemes. In the latter case, the parallel gradient at the $y$ boundary of the system is computed using one-sided derivatives. The value of $q$ has been fixed to $4, N_{z}$ and $N_{y}$ are scanned by maintaining $N_{y}=2 q N_{z}$. The mode considered is a nearly field-aligned mode $n=4, \Delta m=2(m=18)$ mode.

As expected, the $m n$ scheme gives results valid up to machine precision when the number of grid points is large enough to resolve the Fourier harmonics. For the other schemes, the order of accuracy is recovered. The top left panel of Fig. 3 also shows that the fa2 method is more accurate than the $y z 2$ scheme for a periodic geometry while those two methods give equivalent result in non-periodic geometry. Thus, in the periodic case, obtaining an accuracy level equivalent to the fa2 scheme requires a higher resolution and a higher order scheme. In the non-periodic case, using a higher order scheme gives much more accurate results than the fa2 scheme. These findings are amplified if one looks at the accuracy of the $\nabla_{\|}^{2}$ operator for a high $k_{\|}$mode in the bottom right panel of Fig. 3. The following conclusions can be drawn: the alternative scheme to the fa2 scheme requires a higher accuracy order and a higher grid resolution to obtain similar numerical errors. However, at the limiter position, a higher order scheme gives more accurate results compared to the fa2 scheme.

Next, the dispersion relation (38) is compared against numerical results for various spatial discretisation schemes. The physical parameters for this scan are $q=4, m_{i} / m_{e}=200, \eta=5$ and the numerical parameters are $N_{y}=128, N_{z}=32, \Delta t=2 \cdot 10^{-4}$ except for the $f a 2$ scheme where $N_{y}=256$ has to be imposed to have 

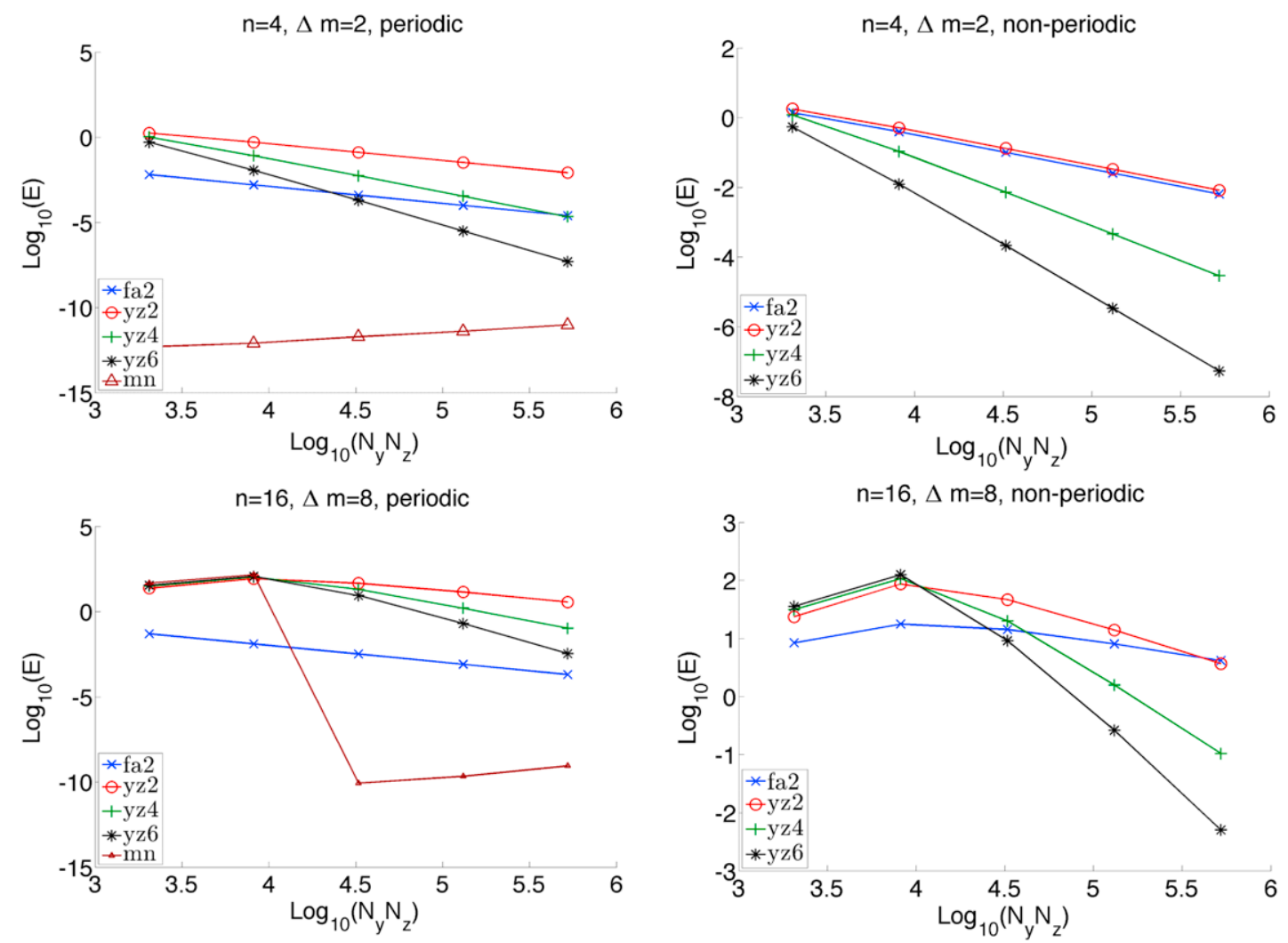

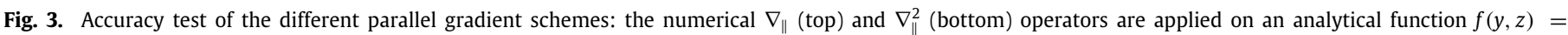

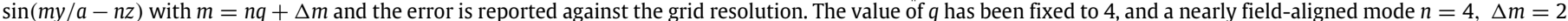

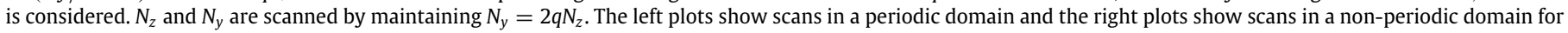
which one sided-derivatives are used.

$\Delta j \geq 2$. Those parameters are rescaled from typical GBS simulations at $L_{y}=800$ [32].

Fig. 4 shows that the $m n$ scheme reproduces the dispersion relation with a very high accuracy as the $m n$ scheme is analytical. Fig. 4 shows the numerical dispersion relation obtained with the fa2 scheme. Results are fairly accurate for $n \leq 4$. For $n>4$, both real frequency and damping rate are not captured, due to the lack of resolution in the $y$ direction. The same exercise shows that the $y z 2$ method is completely inaccurate. On the other hand, the yz4 method, although slightly less accurate, seems to yield comparable results to the fa2 method. The numerical real frequencies and damping rates start to diverge from the analytical value once the poloidal wave number becomes too high with respect to the poloidal resolution. The results of the $y z 6$ scheme (not shown) do not reveal clear signs of improvements compared to the $y z 4$ scheme.

Another useful check consists in studying the behaviour of the eigenmodes of the system, i.e. the $k_{\|}=0$ modes. Physically, such modes should neither oscillate nor be damped. Results are plotted in Fig. 5. When $\eta=0$ (left panel), the eigenmodes of the system exhibit a numerical oscillation that tend to increase with $n$. This oscillation becomes important only at $n>6$ for schemes $y z 2 / y z 4 / y z 6$. Note that there is no oscillation for the $f a 2$ and $m n$ schemes. The right panel shows the numerical damping of $k_{\|}=0$ modes for $\eta=5$.

In summary, various possible schemes to compute the parallel gradient in nonlinear SOL turbulence codes, such as GBS, have been tested using a standalone code for the SAW dynamics and compared against the analytical theory. Results show that an accurate discretisation that does not take advantage of the fieldalignment requires a higher resolution and a higher order of accuracy. The inaccuracy of the schemes become more visible at large $n$. Nevertheless, it is anticipated that these schemes are applicable to SOL turbulence simulations, dominated by low $n$ modes. Based upon the results of the standalone code, we implement the $y z 4$ scheme in GBS and we carry out several tests by using GBS, as described below.

\section{Non-linear tests with GBS}

Several benchmark tests have been carried out to verify that the $y z 4$ scheme can recover previously known results. In order to benchmark a new version of GBS with the $y z 4$ scheme, a scan in $N_{y}$ for a standard electrostatic simulation is presented and these simulations are compared with a simulation using the $f a 2$ scheme. Then, an electromagnetic case showing the transition from resistive to ideal ballooning modes is presented, recovering the ideal threshold found in Ref. [31]. Finally, it is demonstrated that a simulation with a radially varying safety factor profile can be carried out with the $y z 4$ scheme, circumventing the constant- $q$ limitation of the fa2.

\subsection{Benchmark simulations}

According to the results of Section 4, the toroidal resolution for the $y z 4$ simulations should be twice the one needed in a fa2 simulations. This resolution increase introduces unnecessary short wavelengths modes into the system and, according to the CFL condition (42), it decreases the maximum time step. These high$n$ modes are eliminated through Fourier filtering of toroidal modes with $|n|>N_{z} / 4$ at each Runge-Kutta substep. The filtering is applied on all the fluid moments, only for the $y z 4$ scheme. The suppression of these modes allows to relax the CFL condition. 


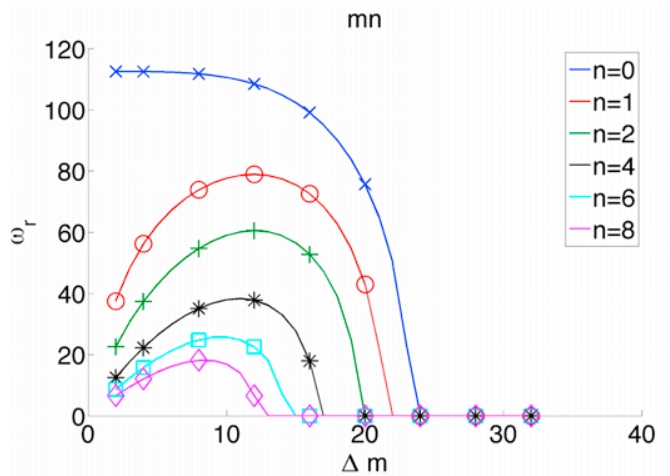

fa
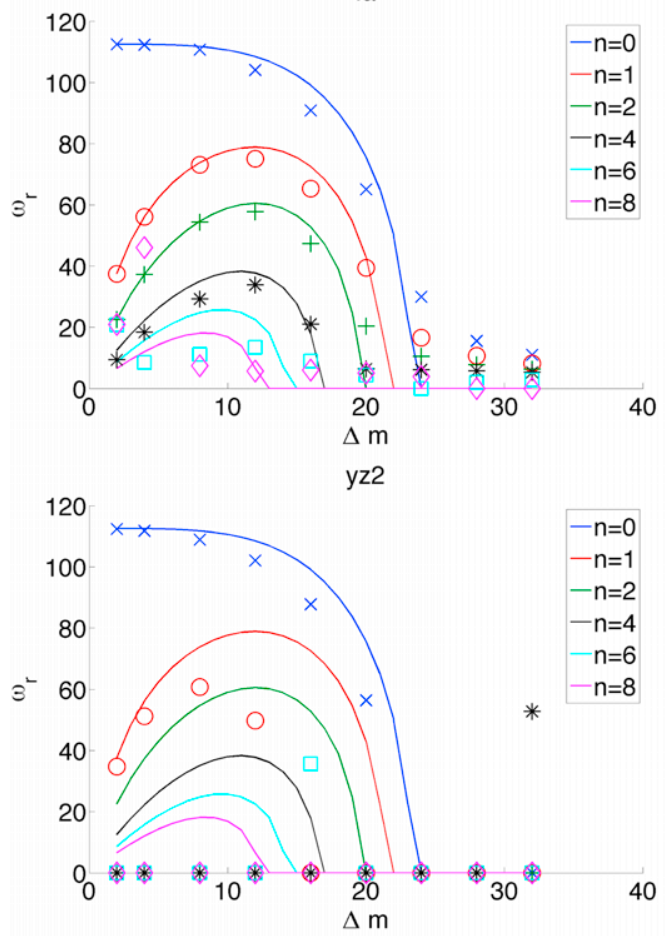

yz4

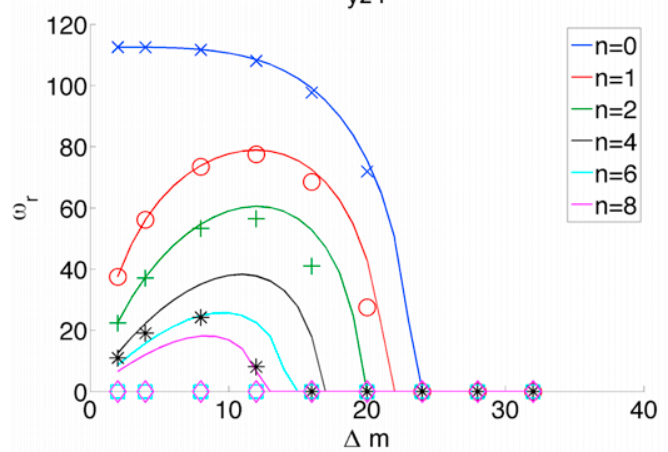

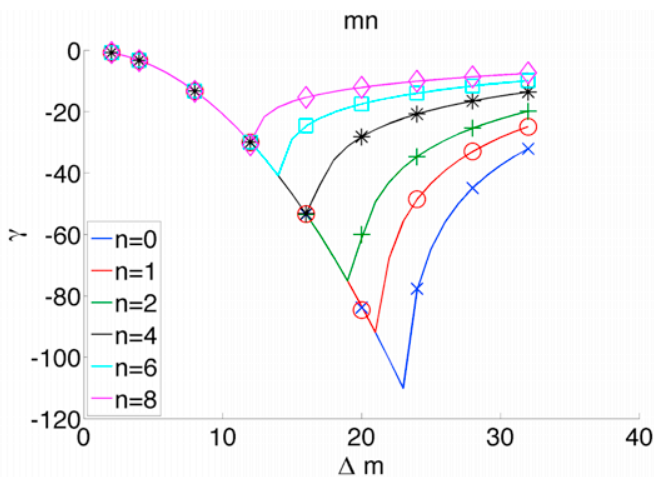

fa
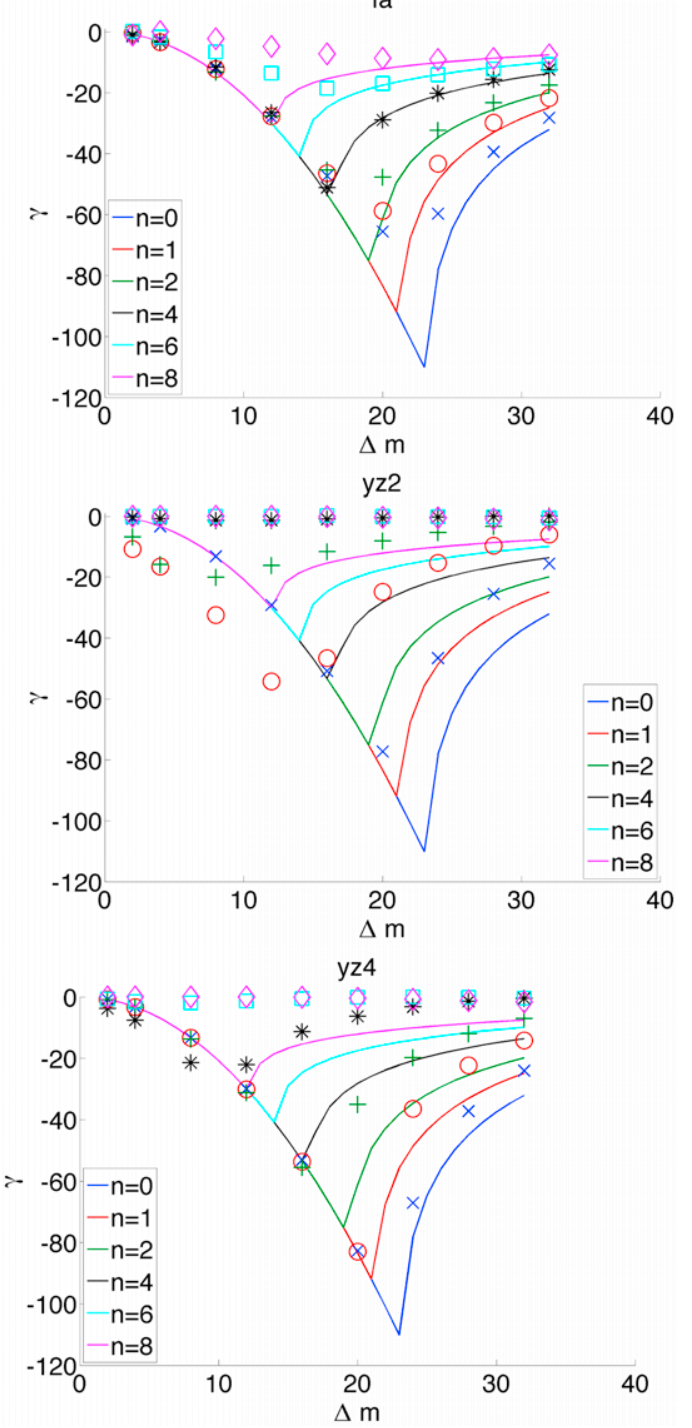

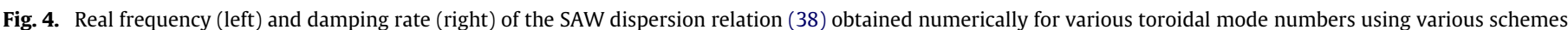

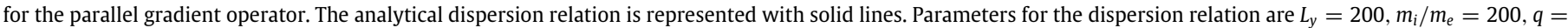
$4, \eta=5, N_{y}=128, N_{z}=32, \Delta t=2 \cdot 10^{-4}$.

Simulations are initialised with radially flat and poloidally smooth profiles consistent with the boundary conditions described in Section 2. A small perturbation is superimposed. After the initial phase of the simulation, the localised injection of density and temperature introduces the free energy necessary to trigger a number of unstable modes that develop into turbulence. The simulations will therefore experience a transient phase followed by a quasi steady-state given by the interplay between the plasma sources, perpendicular transport, and parallel losses at the limiter plates. There is no separation between the background gradient and the fluctuations: the profile gradients are a priori unknown, and are extracted from the time-averaged data over the quasi steady-state.

Simulations with parameters $q=4, \hat{s}=0, L_{x}=100, L_{y}=$ $400, R_{0}=500, v=0.1, m_{i} / m_{e}=200, \eta_{0 e}=\eta_{0 i}=2$ are considered in the large aspect ratio circular magnetic geometry. The 

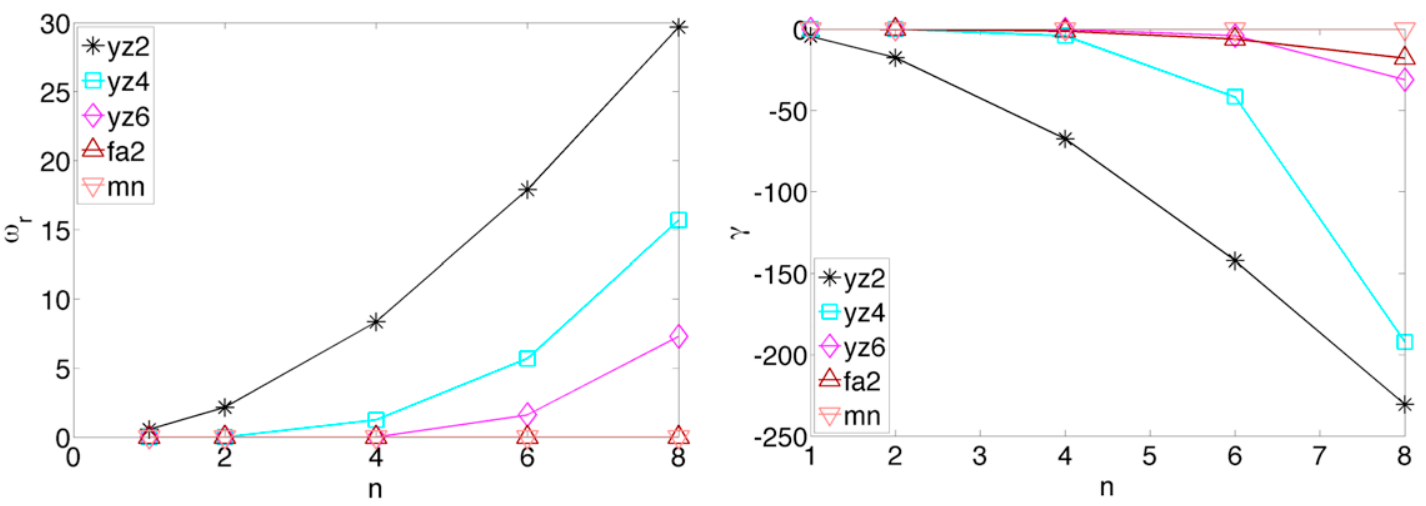

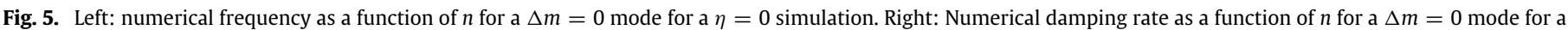
$\eta=5$ simulation. Other parameters are $L_{y}=200, m_{i} / m_{e}=200, q=4, N_{y}=128, N_{z}=32, \Delta t=2 \cdot 10^{-4}$.
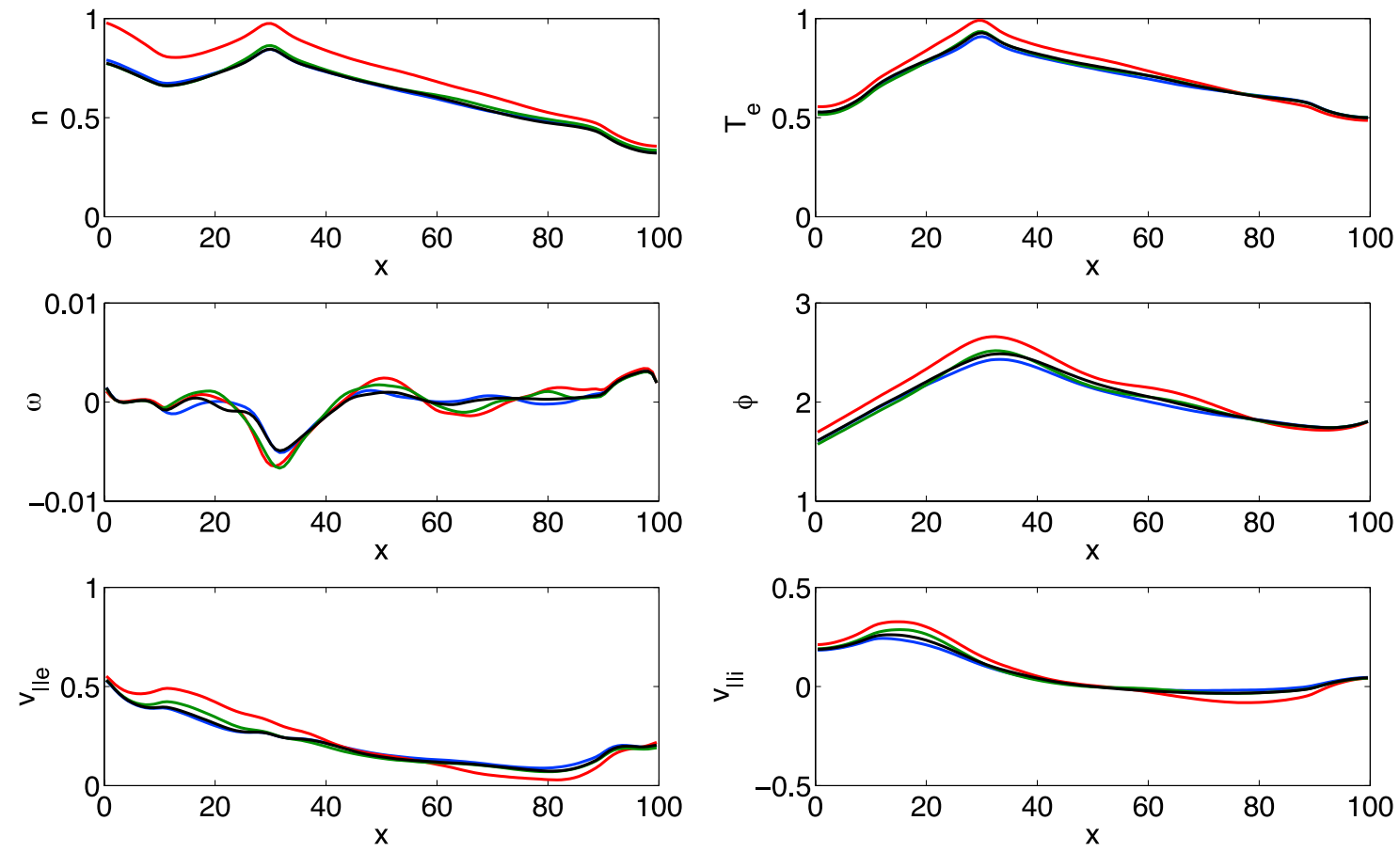

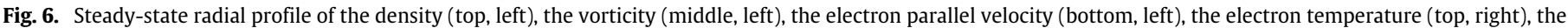

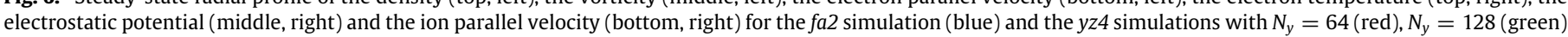

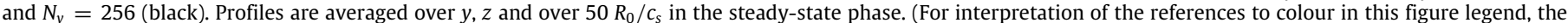
reader is referred to the web version of this article.)

source terms $S_{n}$ and $S_{T_{e}}$ in Eqs. (1) and (5) have a Gaussian shape centred at $x_{S}=30$ with a characteristic width $\sigma_{s}=5$ and have an amplitude of 1 . The source profiles are flat in the $y$ direction but decay exponentially at a distance of $40 \rho_{s}$ from the top and bottom limiter. As these source terms mimic the outflow of plasma from the closed flux surface region, the simulations are physically meaningful for $x>x_{S}$. In a previous work [19], the source strength was varied by a factor of four without significant changes in the dynamics. Simulations with parameters similar to the ones described herein have been used to study, among the others, the mechanisms regulating the turbulent amplitude [19] and the role of EM effects [31]. The following numerical parameters were used for the fa2 simulation: $N_{x}=128, N_{y}=256, N_{z}=32, \Delta t=6 \cdot 10^{-5} R_{0} / c_{s}$. Three $y z 4$ simulations have been performed with the same numerical parameters and $N_{y}=64,128,256$, having doubled the $z$ resolution, i.e. $N_{z}=64$. Figs. 6 and 7 show the radial and poloidal steady-state profiles of these fa2 and $y z 4$ simulations. As GBS simulations are mainly used for the prediction of the radial and poloidal profiles of $n, T_{e}, \phi, v_{\| e}$, and $v_{\| i}$, we focus on these quantities. Clearly, the $y z 4, N_{y}=64$, simulation shows a significative difference with respect to the $f a 2$ profiles. The difference decreases at larger $N_{y}$. The difference between $y z 4$ simulation profiles and the $f a 2$ simulation are below $10 \%$ for $N_{y}=128$, and below $1 \%$ for $N_{y}=256$. The application of the Grid Convergence Index (GCI) [34] to estimate the discretisation error affecting the simulation results show that the quantities that constitute the main interest of GBS simulations are affected by an uncertainty below $5 \%$, for the $y z 4, N_{y}=256$, simulation. As for the vorticity profile of Fig. 7 , note that the advection term in the density and temperature equations involves the potential, which is an integrated quantity with respect to the vorticity. In fact, the vorticity does not enter directly in any of the equations, if not through the potential. The small difference in the potential near the source is amplified when evaluating $\omega=-\nabla^{2} \phi$.

Next, the turbulent properties are compared. Fig. 8 shows the pdf of the potential fluctuations, which looks similar in all cases, with an error bar of about 5\%. The highest resolved cases using the non-field aligned scheme (yz4) fall within this uncertainty compared to the original field aligned parallel gradient scheme 

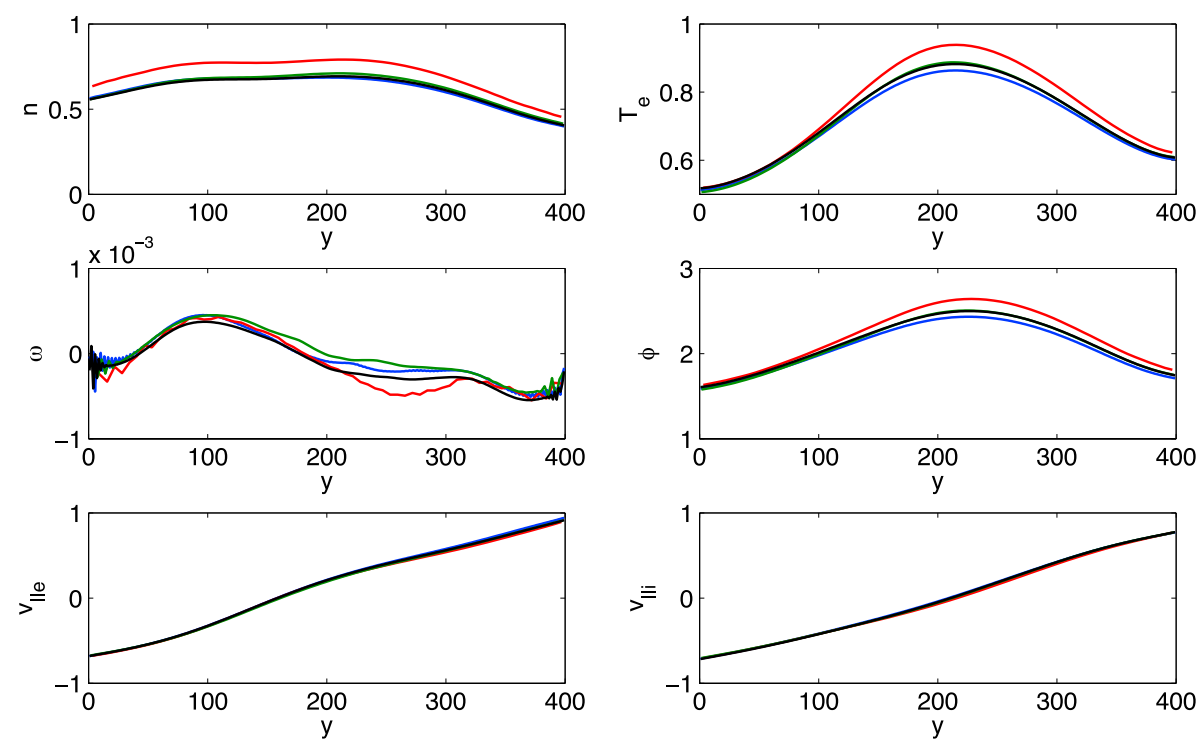

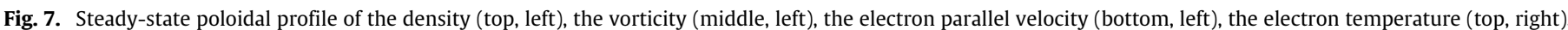

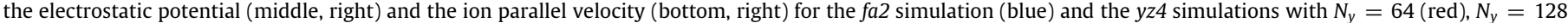

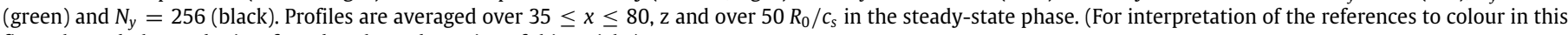
figure legend, the reader is referred to the web version of this article.)

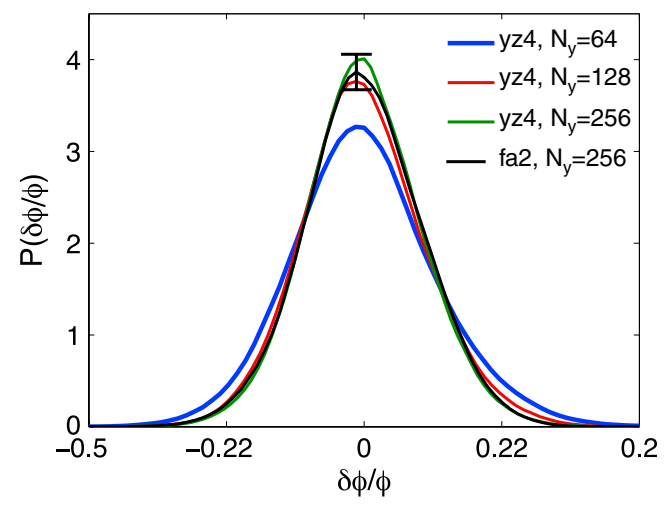

Fig. 8. Distribution function of the perturbed potential for various simulations. The pdfs are computed at $z=0$ for $35 \leq x \leq 80$, the whole poloidal domain and a time window of $50 c_{s} / R$ in the steady-state phase.

(fa2). This points out that the different numerical schemes agree within statistical error. The left panel of Fig. 9 shows the poloidal and toroidal spectrum of the potential in the steady-state phase for the fa2 simulation. Aliasing is clearly visible in the corners at high $|m|$ and $|n|$. These unphysical couplings are suppressed when

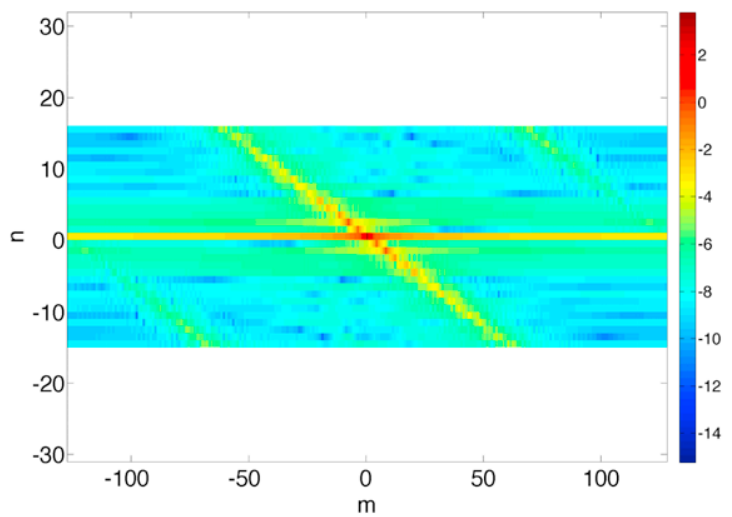

applying the dealiasing procedure in the $y z 4$ simulation. When the $|n|>N_{z} / 4$ fluid moments are filtered out, they are still produced through the numerous nonlinear couplings contained in Eqs. (1)(5). However, their amplitude remains limited as they are filtered at every time step.

\subsection{Electromagnetic simulations}

In this section, nonlinear electromagnetic simulations are carried out using the same parameters as in Section 5.1 except for $q=6, L_{y}=800$. A $\beta_{e}=0$, electrostatic simulation and a $\beta_{e}=6 \cdot 10^{-3}$ electromagnetic simulation have been performed. Electromagnetic effects tend to destabilise ideal ballooning modes (IBM) [31] which are triggered for $\alpha_{\mathrm{MHD}}>0.5$ where $\alpha_{\mathrm{MHD}}=$ $p q^{2} \beta_{e} R /\left(p_{0} L_{p}\right)$. Contrary to resistive ballooning modes (RBM), IBM tend to develop at the longest possible parallel wavelength in the system. Fig. 10 shows a snapshot of the electrostatic potential for the two simulations. The electrostatic simulation is dominated by a $m=12$ poloidal mode, corresponding to a $n=2$ mode in the toroidal direction while the electromagnetic simulation is dominated by a $m=6$ mode, corresponding to the longest fieldaligned parallel wavelength available in the system, a characteristic of ideal ballooning modes. The estimated value of $\alpha_{\mathrm{MHD}}$ for the

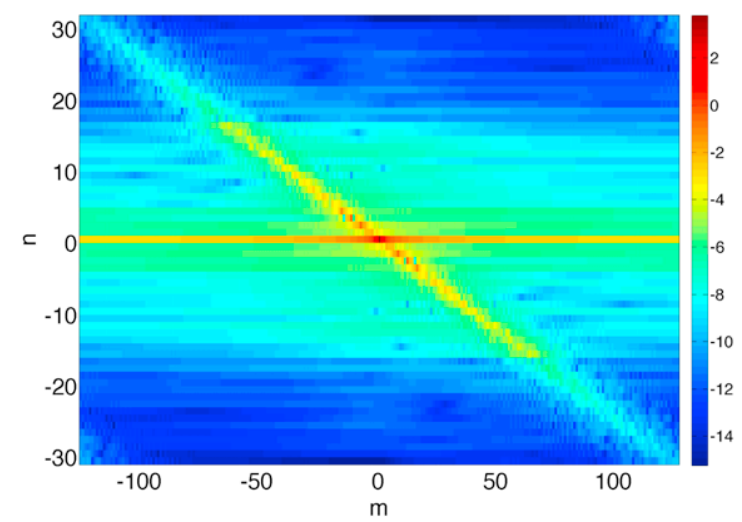

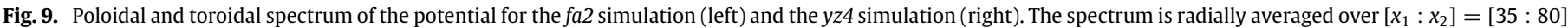
and is time-averaged over $50 R_{0} / c_{s}$ in the steady-state phase. 

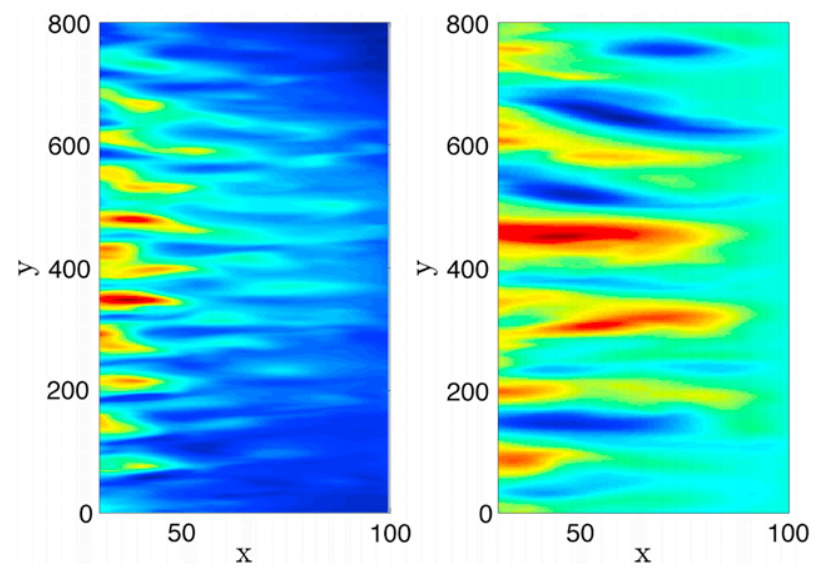

Fig. 10. Snapshot of the electrostatic potential $\phi$ in the poloidal plane for a $\beta_{e}=0$, electrostatic (left) and a $\beta_{e}=6 \cdot 10^{-3}$ electromagnetic simulation. Both simulations have $q=6$.

electromagnetic simulation is 0.6 and exceeds the threshold. The conclusion is that the ideal ballooning threshold, as studied in Ref. [31], can be correctly captured by the $y z 4$ scheme.

\subsection{Global safety profile simulations}

The goal of the present subsection is to demonstrate that the $y z 4$ scheme is able to simulate a SOL with a radially varying safety profile. The implemented profile is:

$q(x)=q_{S}\left(\frac{a+x}{a+x_{S}}\right)^{\hat{s}}$

where $\hat{s}$ is an input parameter representing the magnetic shear and $q_{S}$ is the value of the safety profile at the source position $x_{S}$. This safety profile has a constant shear throughout the SOL. The nonlinear simulations described below have the same parameters as in Section 5.1 with $q_{s}=4$. Simulations at $\hat{s}=-1$ and $\hat{s}=1$ have been carried out.

The effect of a varying $q$ profile on SOL turbulence can be seen in Fig. 11, where the perturbed density is plotted along the $x=80$ magnetic surface for the $\hat{s}=-1,0,1$ simulations. The corresponding safety factor is $q=2.6,4,6.1$, respectively. This plot shows field-aligned structures consistent with the expected pitch of the field line.

Fig. 12 shows the electrostatic potential on the poloidal plane for a given time in the nonlinear steady-state phase. At negative shear, the radial correlation length of the turbulent eddies is strongly reduced, whereas it is amplified at positive shear. In electrostatic simulations, several turbulent regimes may coexist such as resistive ballooning modes, inertial ballooning modes, resistive drift waves or inertial drift waves. The dominant regime depends on the phase space parameters $v, m_{e} / m_{i}, q, \hat{s}$. For the present parameters, it has been shown that the dominant regime at $\hat{s}=0$ is the resistive ballooning mode [32]. At negative shear, resistive ballooning modes tend to be stabilised while they can be destabilised at small positive shear [20]. This trend is confirmed by calculating the sustained pressure gradient by averaging toroidally, poloidally, in time over a steady-state window of $30 R_{0} / C_{s}$, and radially between $x_{s}$ and $x=80$. The values obtained are $R_{0} / L_{p}=$ $30.8,11.6,7.1$ for $\hat{s}=-1,0,1$, respectively. Ballooning modes are strongly suppressed at negative shear, resulting in a much higher sustained pressure gradient inside the SOL. On the other hand, small positive shear destabilises ballooning modes and the sustained pressure gradient decreases.

\section{Conclusion}

In this work, flux-driven 3D fluid simulations of SOL turbulence in limited circular geometry are performed using an approach to the parallel gradient operator based on a non-field-aligned scheme. Precisely, it is shown, through a comparison with the analytical dispersion relation of electrostatic SAWs, that a discretisation using 4th order centred finite differences in both the poloidal and toroidal direction is accurate enough to study the evolution of low wave number modes, provided that the resolution is increased with respect to a field-aligned scheme. This increase of the possible mode spectrum further constrains the CFL condition so that a toroidal mode filter may need to be implemented. This is the case in GBS. Nonlinear electrostatic simulations using this filtering technique and the new parallel gradient scheme have been successfully benchmarked against original GBS simulations. The two cases show good agreement on the equilibria and the fluctuation properties are very similar. Furthermore, a convergence test in the poloidal direction shows that the resolution employed is sufficient. The CPU cost of the non-field-aligned simulation is slightly
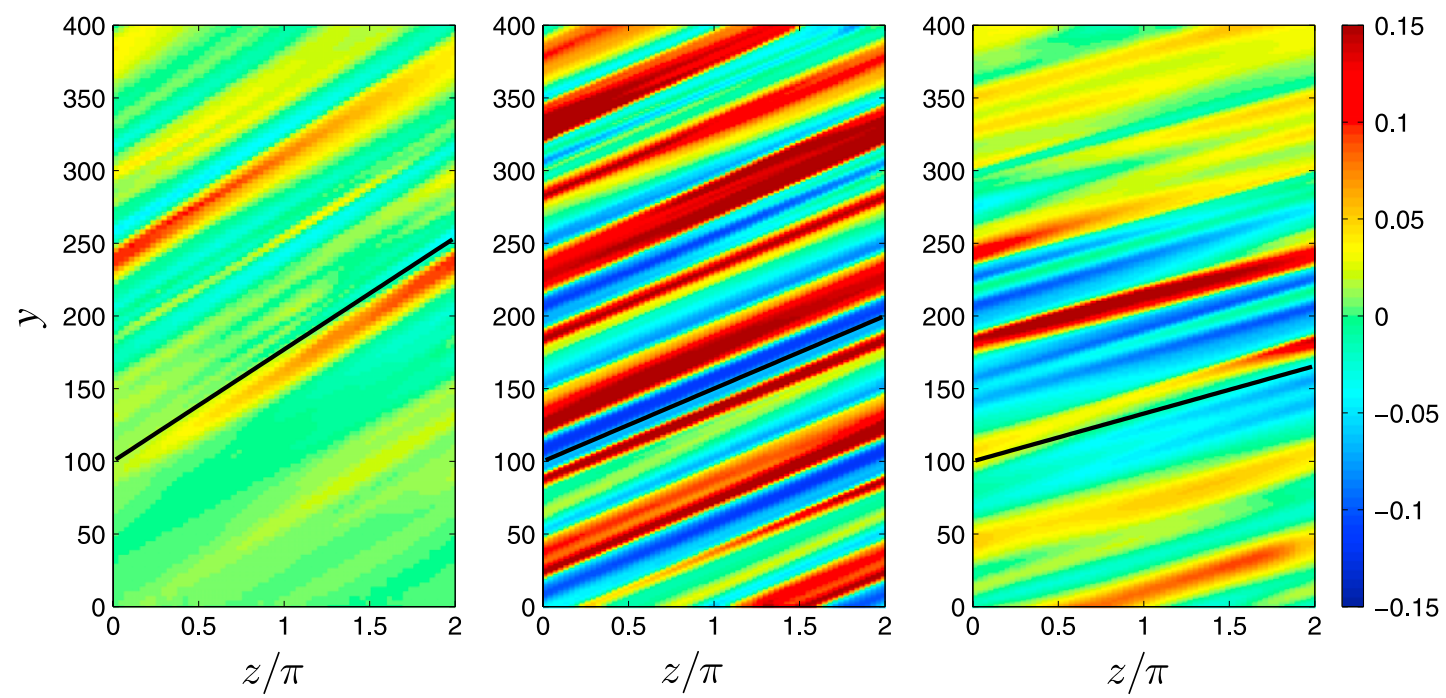

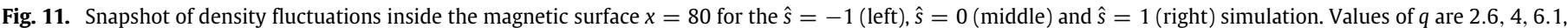
respectively. The superimposed black lines indicate the pitch of the magnetic field lines. 

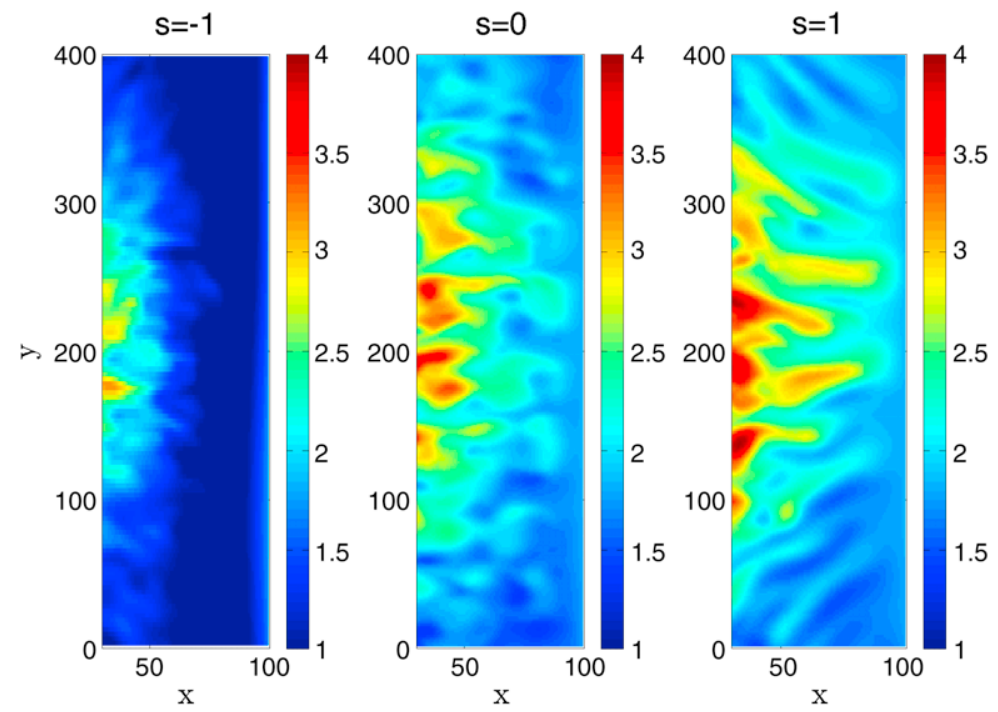

Fig. 12. Snapshot of the electrostatic potential in the poloidal plane for the $\hat{s}=-1$ (left), $\hat{s}=0$ (middle) and $\hat{s}=1$ (right) simulation.

increased with respect to the field-aligned one. The new scheme is also successfully applied to electromagnetic simulations, where the presence of an ideal ballooning mode is retrieved. The new scheme is applied to a configuration with a radially varying safety profile and the RBM stabilisation at negative shear is recovered.

More generally, the present work shows that it is possible to simulate SOL turbulence without a field-aligned approach at a reasonable cost. The main reason is that turbulence in this region is dominated by low wavenumber modes that are accurately resolved by a non-field-aligned scheme.

\section{Acknowledgments}

The simulations presented herein were carried out at the Swiss National Supercomputing Centre (CSCS), under project ID s346, and at the Computational Simulation Centre of the International Fusion Energy Research Centre (IFERCCSC), Aomori, Japan, under the Broader Approach collaboration between Euratom and Japan, implemented by Fusion for Energy and JAEA. This work was supported by the Swiss National Science Foundation and by the Eurofusion Enabling Research program.

\section{References}

[1] S. Ku, C.S. Chang, P.H. Diamond, Nucl. Fusion 49 (2009) 115021

[2] C.S. Chang, S. Ku, P.H. Diamond, Z. Lin, S. Parker, T.S. Hahm, N. Samatova, Phys. Plasmas 16 (2009) 056108

[3] D.P. Stotler, C.S. Chang, S.H. Ku, J. Lang, G.Y. Park, J. Nucl. Mater. 438 (2013) S1275.

[4] O.E. Garcia, V. Naulin, A.H. Nielsen, J.J. Rasmussen, Phys. Rev. Lett. 92 (2004) 165003

[5] Y. Sarazin, P. Ghendrih, G. Attuel, C. Clement, X. Garbet, V. Grandgirard, M Ottaviani, S. Benkadda, P. Beyer, N. Bian, C. Figarella, J. Nucl. Mater. 313 (2003) 796.

[6] M.A. Beer, S.C. Cowley, G.W. Hammett, Phys. Plasmas 2 (1995) 2687.
[7] M. Kotschenreuter, G. Rewoldt, W.M. Tang, Comput. Phys. Comm. 88 (1995) 128.

[8] F. Jenko, Comput. Phys. Comm. 125 (2000) 196

[9] B.D. Scott, Plasma Phys. Control. Fusion 39 (1997) 1635.

[10] B.D. Scott, Phys. Plasmas 8 (2001) 447.

[11] S. Jolliet, A. Bottino, P. Angelino, R. Hatzky, T.M. Tran, B.F. McMillan, O. Sauter K. Appert, Y. Idomura, L. Villard, Comput. Phys. Comm. 177 (2007) 409.

[12] S. Jolliet, B.F. McMillan, L. Villard, T. Vernay, P. Angelino, T.M. Tran, S. Brunner, A. Bottino, Y. Idomura, J. Comput. Phys. 231 (2011) 745.

[13] F. Hariri, M. Ottaviani, Comput. Phys. Comm. 184 (2013) 2419.

[14] S. Guenter, Q. Yu, J. Krueger, K. Lackner, J. Comput. Phys. 209 (2005) 354.

[15] S. Guenter, K. Lackner, C. Tichmann, J. Comput. Phys. 226 (2007) 2306.

[16] Y. Idomura, H. Urano, N. Aiba, S. Tokuda, Nucl. Fusion 49 (2009) 065029.

[17] P. Tamain, P. Ghendrih, E. Tsitrone, V. Grandgirard, X. Garbet, Y. Sarazin, E. Serre, G. Ciraolo, G. Chiavassa, J. Comput. Phys. 229 (2010) 361.

[18] P. Ricci, F.D. Halpern, S. Jolliet, J. Loizu, A. Mosetto, A. Fasoli, I. Furno, C. Theiler, Plasma Phys. Control. Fusion 54 (2012) 124047.

[19] P. Ricci, B.N. Rogers, Phys. Plasmas 20 (2013) 010702.

[20] A. Mosetto, F.D. Halpern, S. Jolliet, J. Loizu, P. Ricci, Phys. Plasmas 20 (2013) 092308

[21] F.D. Halpern, P. Ricci, B. Labit, I. Furno, S. Jolliet, J. Loizu, A. Mosetto, G. Arnoux J.P. Gunn, J. Horacek, M. Kocan, B. LaBombard, C. Silva, J.E. Contributors, Nucl. Fusion (2013)

[22] J. Loizu, P. Ricci, F.D. Halpern, S. Jolliet, A. Mosetto, Plasma Phys. Control. Fusion 55 (2013) 124019

[23] A. Zeiler, D. Biskamp, J.F. Drake, P.N. Guzdar, Phys. Plasmas 3 (1996) 2951.

[24] J. Loizu, P. Ricci, F.D. Halpern, S. Jolliet, Phys. Plasmas 19 (2012) 122307.

[25] B.D. Dudson, M.V. Umansky, X.Q. Xu, P.B. Snyder, H.R. Wilson, Comput. Phys. Comm. 180 (2009) 1467.

[26] A. Thyagaraja, Plasma Phys. Control. Fusion 42 (2000) B255.

[27] V. Naulin, Phys. Plasmas 10 (2003) 4016.

[28] D. Reiser, Phys. Plasmas 14 (2007) 082314.

[29] B.D. Scott, Plasma Phys. Control. Fusion 45 (2003) A385.

[30] A. Arakawa, J. Comput. Phys. 1 (1966) 119.

[31] F.D. Halpern, S. Jolliet, J. Loizu, A. Mosetto, P. Ricci, Phys. Plasmas 20 (2013) 052306.

[32] S. Jolliet, F.D. Halpern, J. Loizu, A. Mosetto, P. Ricci, Phys. Plasmas 21 (2014) 022303.

[33] W.W. Lee, J.L.V. Lewandowski, T.S. Hahm, Z. Lin, Phys. Plasmas 8 (2001) 4435

[34] P.J. Roache, J. Fluids Eng. 116 (1994) 405 